\title{
A ATITUDE TRANSDISCIPLINAR APLICADA A SAÚDE E SUSTENTABILIDADE, UMA ABORDAGEM MULTIDIMENSIONAL: A IMPORTÂNCIA DA MEDITAÇÃO
}

\author{
ACTITUD TRANSDISCIPLINARIA PARA LA SALUD Y LA SOSTENIBILIDAD, \\ UN ENFOQUE MULTIDIMENSIONAL: \\ LA IMPORTANCIA DE LA MEDITACIÓN
}

\section{ATTITUDE TRANSDISCIPLINARY APPLIED HEALTH AND SUSTAINABILITY, A MULTIDIMENSIONAL APPROACH: THE IMPORTANCE OF MEDITATION}

BIGNARDI, Fernando A. C. ${ }^{1}$

RESUMO: Diante do aumento demográfico da população idosa no mundo, constatamos que a medicina fundamentada numa concepção mecânica de homem não se mostra suficiente e satisfatória. Por ser um método topográfico, que é acionado quando o médico identifica qual a parte doente do corpo, ao que se segue um diagnóstico e consequentemente um tratamento, decorre assim uma somatória de prescrições diante da multiplicidade de diagnósticos. Como a maioria dos idosos tem mais de um diagnóstico (75\%), ocorre o sério problema da polifarmácia (como é conhecida a sobreposição de vários medicamentos simultâneos em um mesmo indivíduo), cujos efeitos colaterais levam à internação hospitalar cerca de $12 \%$ dos idosos tratados. Além deste índice de internações, $80 \%$ dos idosos tratados pela medicina convencional têm pelo menos um efeito colateral em seu tratamento! O estudo epidemiológico sobre o envelhecimento demonstra que a principal causa de perdas funcionais na velhice, em decorrência das doenças crônicas, é o estilo de vida, que resulta de fatores multidimensionais, como escolhas alimentares, hábitos de sono e ritmo de vida, crenças, postura e atividade física etc. Desta forma, mudar do modelo mecânico newtoniano, adotado pela Medicina Convencional, desde a perseguição aos pensadores sistêmicos realizada pela Inquisição no final do primeiro milênio, para uma concepção multidimensional inspirada na física quântica contemporânea, foi fundamental para compreender melhor o processo de adoecer do ser humano. Na concepção multidimensional, a qual considera as dimensões: física (órgãos e sistemas), metabólica (fluido composto por sangue e linfa que integra e comunica os sistemas), vital (relacionado aos ritmos do corpo: sono, apetite, excreções, respiração etc.) mental (postura física e atitude mental diante da realidade interna e externa) e supramental (espiritualidade como "arquivo" da missão essencial e da Individualidade do ser humano), pode-se identificar uma cascata de causalidade descendente (5) que se inicia quando a pessoa se desconecta de sua vocação essencial (dimensão

\footnotetext{
${ }^{1}$ Médico gerontólogo, psicoterapeuta, homeopata e consultor pessoal e corporativo na área da sustentabilidade. Coordenador do Centro de Estudos do Envelhecimento da UNIFESP e diretor fundador do Centro de Ecologia

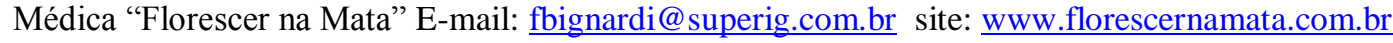


supramental ou espiritual) e constrói modelos mentais de vida ancorados numa percepção ilusória da realidade, com valores circunstanciais como a ambição material baseada no poder alcançado pela violência, conhecido como Androcracia. Esta atitude de exploração baseada no desrespeito pelo outro e na dominação da Natureza é a principal causa da insustentabilidade que aflige o mundo contemporâneo, com reflexos diretos na saúde.

Palavras chave: saúde, sustentabilidade, transdisciplinaridade, meditação, abordagem multidimensional

RESUMEN: El aumento demográfico de la población de edad avanzada en el mundo, ha nos mostrado que la medicina basada en una concepción mecanicista del hombre no se muestra suficiente y satisfactorio. Siendo un método topográfico, cuando el médico identifica la parte enferma del cuerpo, después de un diagnóstico y tratamiento por lo tanto, surge una suma de medicamentos antes de que la multiplicidad de diagnósticos. Como la mayoría de las personas mayores tienen más de un diagnóstico $(75 \%)$, hay un problema grave de la polifarmacia (conocida como la superposición de múltiples fármacos simultáneamente en el mismo individuo), cuyos efectos secundarios han llevado a la hospitalización aproximadamente 12\% de los ancianos tratada. Además de este índice de hospitalización, $80 \%$ de las personas de edad tratados por la medicina convencional tiene al menos un efecto secundario en su tratamiento! El estudio epidemiológico en los programas de envejecimiento ha demostrado que la causa principal de la pérdida funcional en la vejez, como consecuencia de las enfermedades crónicas, es el estilo de vida resultante de factores multidimensionales, tales como la elección de alimentos, hábitos de sueño y su estilo de vida, creencias, actitudes y la actividad física etc. Por lo tanto, cambiar del modelo newtoniano mecánico adoptado por la medicina convencional, ya que la persecución de los pensadores sistémicos en poder de la Inquisición a finales del primer milenio, para un diseño contemporáneo multidimensional inspirado en la física cuántica es esencial para comprender mejor el proceso de enfermedad humano. En concepto multidimensional, que considera dimensiones: físicas (órganos y sistemas), metabólicas (líquido compuesto de la sangre y de la linfa que integra y comunica los sistemas), vital (en relación con los ritmos del cuerpo: el sueño, el apetito, la excreción, respiración etc.) mental (postura física y la actitud mental frente a la realidad interna y externa) y supramental (espiritualidad como "archivo" misión esencial y la individualidad del ser humano), se puede identificar una cascada de causalidad descendente (5) que se inicia cuando una persona se desconecta de su vocación esencial (o dimensión espiritual supramental) y construir modelos mentales de la vida anclada en la percepción ilusoria de la realidad, con valores como la ambición material circunstancial basada en el poder logrado a través de la violencia, conocido como Androcracia. Esta actitud de explotación basada en la falta de respeto por los demás y en el dominación de la naturaleza es la causa principal de insostenibilidad que aflige al mundo contemporáneo.

Palabras clave: la salud, la sostenibilidad, la transdisciplinaridad, la meditación, el enfoque multidimensional.

ABSTRACT: Given the increasing population of the elderly population in the world, we find that medicine based on a mechanical conception of man does not show sufficient and satisfactory. Being a topographic method, which is triggered when the doctor identifies which the diseased part of the body, following a diagnosis and therefore treatment, thus arises a sum of prescriptions before the multiplicity of diagnoses. Like most seniors have more than one diagnosis $(75 \%)$, there is a serious problem of polypharmacy (known as the overlap of 
multiple drugs simultaneously in the same individual), whose side effects have led to hospitalization about $12 \%$ of the elderly treated. Besides this index hospitalization, $80 \%$ of the elderly treated by conventional medicine have at least one side effect on your treatment! The epidemiological study about aging shows that the main cause of functional loss in old age, as a result of chronic diseases, it is the lifestyle that results from multidimensional factors such as food choices, sleep habits and lifestyle, beliefs, attitude and physical activity, etc.. Thus, changing the Newtonian mechanical model adopted by Conventional Medicine, since the persecution of systemic thinkers held by the Inquisition in the late first millennium, for a multidimensional design inspired by contemporary quantum physics was essential to better understand the disease process of humans. In multidimensional concept, which considers dimensions: physical (organs and systems), metabolic (fluid composed of blood and lymph that integrates and communicates systems), vital (related to the rhythms of the body: sleep, appetite, excretion, respiration, etc. .) mental (physical posture and mental attitude in the face of internal and external reality) and supramental (spirituality as "file" mission essential and the Individuality of the human being), we can identify a cascade of downward causation (5) that starts when a person disconnects from its essential vocation (or supramental spiritual dimension) and build mental models of life anchored in illusory perception of reality, with values like ambition circumstantial stuff based on power achieved through violence, known as Androcracia. This attitude of exploitation based on disrespect for each other and the domination of nature is the main cause of unsustainability that afflicts the contemporary world.

Key Word: health, sustainability, transdisciplinarity, meditation, multidimensional approach.

\section{INTRODUÇÃO}

Diante do aumento demográfico da população idosa no mundo, constatamos que a medicina baseada numa concepção mecânica de homem não se mostra suficiente e satisfatória. Por ser um método topográfico, que é acionado quando o médico identifica qual a parte doente do corpo, ao que se segue um diagnóstico e consequentemente um tratamento, decorre assim uma somatória de prescrições diante da multiplicidade de diagnósticos.(1)

Como a maioria dos idosos tem mais de um diagnóstico (75\%), ocorre o sério problema da polifarmácia (como é conhecida a sobreposição de vários medicamentos simultâneos em um mesmo indivíduo), cujos efeitos colaterais levam a internação hospitalar cerca de $12 \%$ dos idosos tratados. Além deste índice de internações, $80 \%$ dos idosos tratados pela medicina convencional, têm pelo menos 1 efeito colateral em seu tratamento!(4)

Além disso, o estudo epidemiológico sobre o envelhecimento demonstra que a principal causa de perdas funcionais na velhice, em decorrência das doenças crônicas, é o estilo de vida, que resulta de fatores multidimensionais como escolhas alimentares, hábitos de sono e ritmo de vida, crenças, postura e atividade física, etc. $(2 ; 3)$ 
Em função disso, mudar do modelo mecânico newtoniano, adotado pela Medicina Convencional, desde a perseguição aos pensadores sistêmicos realizada pela Inquisição no final do primeiro milênio, para uma concepção multidimensional inspirada na física quântica contemporânea, foi fundamental para compreender melhor o processo de adoecer do ser humano (5).

Neste modelo, que considera as dimensões: física (órgãos e sistemas), metabólica (fluido composto por sangue e linfa que integra e comunica os sistemas), vital (relacionado aos ritmos do corpo: sono, apetite, excreções, respiração, etc.) mental (postura física e atitude mental diante da realidade interna e externa) e supramental (espiritualidade como "arquivo" da missão essencial e da Individualidade do ser humano), pode-se identificar uma cascata de causalidade descendente (5) que se inicia quando a pessoa se desconecta de sua vocação essencial (dimensão supramental ou espiritual) e constrói modelos mentais de vida ancorados numa percepção ilusória da realidade com valores circunstanciais como a ambição material baseada no poder alcançado pela violência, conhecido como Androcracia. Esta atitude de exploração baseada no desrespeito pelo outro e na dominação da Natureza é a principal causa da insustentabilidade que aflige o mundo contemporâneo (6).

Ao recorrermos a um protocolo terapêutico que valoriza e intervém em todas estas dimensões são criadas as condições para que a cura aliada ao bem estar sejam despertadas na pessoa (7). Em ensaios clínicos corporativos realizados utilizando-se este procedimento multidimensional obtivemos a cura de doenças crônicas decorrente da transformação de padrões patogênicos nos vários níveis da pessoa (8).

Quando tomamos como exemplo a Hipertensão Arterial Sistêmica Essencial (HA), assim chamada porque afeta a totalidade da economia do organismo sem que se encontre um distúrbio anatômico para justificá-la (=essencial), detectamos claramente o fenômeno da causalidade descendente atuando.

Num ensaio clínico realizado numa grande organização brasileira do comércio varejista de eletrodomésticos, onde as metas de venda acabam sendo interpretadas pelos gerentes de venda como fonte de pressão psicológica, pôde-se verificar que a atitude mental associada à HA era de preocupação fazendo com que o trabalhador mantivesse sua atenção mental focada no futuro, alienando-se do presente e de suas necessidades essenciais atuais.

Esta situação acarretava um distúrbio postural de anteriorização em relação ao eixo da gravidade (inclinação para frente de todo o corpo) numa atitude física de prontidão para a ação. 
A vitalidade destas pessoas se encontrava severamente comprometida com prevalência aumentada de insônia, com o sono interrompido e não reparador. O ritmo da vida apresentava-se distorcido: a respiração, normalmente diafragmática e em 4 tempos (inspiração, pausa inspiratória, expiração, pausa expiratória), apresentava-se curta e sem pausas. Existem evidências científicas que a correção artificial do ritmo respiratório melhora a HA. $(9 ; 10)$ Também o apetite e o atendimento às necessidades fisiológicas (intestino preso) estavam afetados.

O metabolismo apresentava-se alterado, com altos índices plasmáticos de homocisteína e ferritina decorrentes de uma obstinada e ansiosa ingestão de carne vermelha associada a um hábito alimentar pervertido.

Este complexo quadro sistêmico culminava com o surgimento da doença cardiovascular crônica.

Observa-se assim, como o estilo de vida e as escolhas cotidianas, se convertem em doença crônica, como revelam as pesquisas epidemiológicas sobre o envelhecimento $(1,2$, $3)$.

Para intervir de forma eficaz neste quadro, necessita-se de uma ferramenta como a meditação, entendida como uma metodologia científica de mudança voluntária do modo de operação cerebral, reconhecida desde os anos 60.

No estado de consciência meditativo, as ondas eletroencefalográficas beta, do estado de vigília habitual, sedem lugar para ondas teta, delta e gama, características do sono profundo, que passam a ocorrer em estado de alerta possibilitando a reconexão do praticante com as dimensões causais da realidade representadas por uma sutil rede de padrões sistêmicos que formata, por meio de informações, a matéria e a energia $(11 ; 12)$.

Esta condição já era referida na Bíblia quando se refere ao Verbo que existia no princípio e se fez carne, ao mesmo tempo em que relata que Deus disse (emitiu uma informação): Faça-se a luz!

A mente meditativa se conecta ao universo simbólico reconhecendo imagens carregadas de significado. Essa constatação sugere a compreensão do mecanismo de ação dos procedimentos que se valem de técnicas imaginativas (como as visualizações), que costumam ser tão benéficas no resgate da saúde. A consultora de manejo animal Temple Grandin relata que sua mente autista "pensa" em imagens, assim como fazem os animais, e, desta forma, consegue compreendê-los e se comunicar com eles (13). Penélope Smith confirma a importância da mente aquietada e das imagens na comunicação com os animais (14), assim 
como a equitadora Sylvana Smith em seu artigo "The Mental connection" (15), ressalta a importância de se comunicar com os cavalos por meio de imagens e não frases mentais.

Nosso cérebro é equipado com dispositivos de acesso a rede de padrões informacionais sistêmicos, preconizados pelo físico austríaco Fritjof Capra como o sustentáculo da sustentabilidade universal (16), estudos realizados com SPECT (Single Photon Emission Computed Tomography) por Dr. Andrew Newberg e Eugene D'Aquili, evidenciam que o hemisfério direito se ativa no momento em que meditadores experientes entram em estado de profunda quietude e silêncio, conectando-se com o que poderíamos chamar de plano essencial. A neurocientista americana, Jill Taylor, ao se recuperar de um acidente vascular encefálico que afetou seu hemisfério esquerdo, pode experimentar a plenitude das funções cerebrais do hemisfério direito e as descreve em detalhes no seu livro (17).

Este processo de reconexão do ser humano com esta dimensão espiritual ou causal da Realidade possibilita o realinhamento de alguém com sua vocação essencial alinhada com o projeto cósmico de realização universal que resulta em ações sustentáveis, como se constatou nos resultados obtidos pelo Programa de Desenvolvimento Humano realizado no SEBRAEMT em 2008, utilizando-se, além de recursos como a meditação o método fenomenológico de Goethe, que possibilita ao praticante, o acesso à rede informacional que dá suporte a realidade concreta.

Esta condição de sintonia era, como sugerem evidencias arqueológicas obtidas na Grécia, época cultural conhecida como Europa Antiga e na Amazônia (18,19), a estrutura cultural das civilizações primitivas, onde o espírito feminino de parceria entre ser humano e Natureza prevalecia e possibilitou o sucesso da espécie na sua jornada de sobrevivência no Planeta por centenas de milhares de anos.

É curioso que esta fase primeva de liderança feminina é lembrada na tradição oral das tribos amazônicas!

O resgate da cultura de parceria apresenta-se como um pilar fundamental na reconquista da sustentabilidade pessoal e planetária. Para tanto a contemplação dos fenômenos e tomada de decisões ancoradas nas funções do hemisfério encefálico direito (acessado pela meditação e outras técnicas contemplativas como as utilizadas na fenomenologia goethiana) apresenta-se como etapa fundamental do resgate da saúde, bem estar, realização e cultura de paz e prosperidade. 
Uma forte evidência deste fenômeno é observada em grupos de idosos que meditam, nos quais a reconexão com a dimensão supramental, viabilizada pela meditação, favorece o resgate da missão essencial da vida e possibilita o ressurgimento da motivação pela vida acompanhada da cura de doenças crônicas (por exemplo: hipertensão arterial, diabetes e até glaucoma!), assim como o resgate do bem estar e qualidade de vida na medida em que são retomados, e realizados, antigos projetos de vida.

Esta cascata de causalidade, da dimensão supramental até a física foi demonstrada pelo ensaio clínico realizado em 2010/2011 com cerca de 140 idosos do bairro de São Matheus (periferia da cidade de São Paulo) no qual, os participantes voluntários foram treinados em meditação e convidados a praticá-la em 2 períodos de no mínimo de 20 minutos cada vez, ao dia.

O ensaio se deu com dois grupos de 70 participantes, um em 2010 e o outro em 2011. Os resultados foram compilados em 2 subgrupos: Os que declararam meditar pelo menos uma vez ao dia e os que praticavam apenas uma vez por semana no encontro coletivo realizado no auditório do Hospital Geral de São Matheus.

As tabelas abaixo sintetizam os resultados obtidos por meio de entrevistas individuais realizadas por profissionais de saúde treinados, aplicando um questionário padronizado.

Os efeitos se referem ao percentual de participantes que apresentaram melhora nos itens relativos a cada uma das dimensões estudadas:

Mental: memória e humor

Transição Mental/Vital: postura

Vital: respiração, sono, hábito intestinal, disposição

Metaloólico: hábito alimentar

Físico: melhora clínica da dor e de doenças crônicas 
1ํ subgrupo: Meditaram no mínimo uma vez ao dia

1 Grupo 2010

\begin{tabular}{|l|l|}
\hline MEMÓRIA & $18(30,51 \%)$ \\
\hline HUMOR & $42(71,19 \%)$, \\
\hline POSTURA & $42(71,19 \%)$, \\
\hline RESPIRAÇÃO & $38(64,41 \%)$, \\
\hline SONO & $34(57,63 \%)$, \\
\hline HÁBITO INTESTINAL & $22(37,29 \%)$, \\
\hline DISPOSIÇÃO & $37(62,71 \%)$, \\
\hline ALIMENTACÇÃO & $34(57,63 \%)$, \\
\hline DORES FÍSICAS & $34(57,63 \%)$ \\
\hline DOENÇAS CRÔNICAS & $27(45,76 \%)$, \\
\hline
\end{tabular}

2음 Grupo 2011

\begin{tabular}{|l|l|}
\hline MEMÓRIA & $18(44,79 \%)$ \\
\hline HUMOR & $27(75,86 \%)$ \\
\hline POSTURA & $37(83,63 \%)$ \\
\hline RESPIRAÇÃO & $34(76,60 \%)$ \\
\hline SONO & $25(69,55 \%)$ \\
\hline HÁBITO INTESTINAL & $16(45,47 \%)$ \\
\hline DISPOSIÇÃO & $26(72,71 \%)$ \\
\hline ALIMENTAÇÃO & $16(42,93 \%)$ \\
\hline DORES FÍSICAS & $11(36,66 \%)$ \\
\hline DOENÇAS CRÔNICAS & $23(56,96 \%)$ \\
\hline
\end{tabular}

É possível observar que os efeitos registrados seguem a cascata de causalidade: partindo da dimensão mais sutil (mental/psiquismo) para a mais densa (física).

Estes dados são corroborados pelos depoimentos dos participantes, que referiam comentários dos familiares e amigos sobre mudanças ocorridas nos comportamentos, crenças, hábitos e atitudes sociais.

A postura física tornou-se mais ereta sem que tenha ocorrido intervenção fisioterápica repercutindo na melhora das dores de origem postural.

No campo da vitalidade o sono melhora e torna-se reparador, alem de ter ocorrido melhora do hábito intestinal e da disposição para as atividades cotidianas.

A população estudada era predominantemente oriunda do Nordeste brasileiro, onde o hábito alimentar carnívoro predomina. Vários depoimentos revelaram que a atitude à mesa se transformou espontaneamente, aumentando a ingestão de alimentos de origem vegetal (cereais, verduras, legumes e frutas) acompanhado do decréscimo na ingestão dos de origem animal (especialmente carne vermelha).

Finalmente, a "onda de causalidade" alcança a dimensão Física reduzindo a incidência de doenças crônicas como o diabetes: participantes referiam que se sentiam tontos quando faziam uso dos hipoglicemiantes orais que estavam habituados, demandando uma redução, e eventual interrupção do uso dos recursos farmacológicos, já que a glicemia havia se normalizado. Resultados semelhantes foram registrados diante da hipertensão arterial e até 
mesmo do glaucoma, dentre outras situações como distimia, depressão, ansiedade, insônia, etc.

2ํsubgrupo: Meditaram apenas uma vez na semana

1․ Grupo: 2010

2ㅇ Grupo: 2011

\begin{tabular}{|l|l|}
\hline MEMÓRIA & -- \\
\hline HUMOR & $3(27,27 \%)$ \\
\hline POSTURA & $4(36,36 \%)$ \\
\hline RESPIRAÇÃO & $4(36,36 \%)$ \\
\hline SONO & $3(27,27 \%)$ \\
\hline HÁBITO INTESTINAL & $2(18,18 \%)$ \\
\hline DISPOSIÇÃO & ---- \\
\hline ALIMENTAÇÃOO & $5(45,45 \%)$ \\
\hline DORES FISICASAS & $1(9,09 \%)$ \\
\hline DOENÇAS CRÔNICAS & ---- \\
\hline
\end{tabular}

\begin{tabular}{|l|l|}
\hline MEMÓRIA & $2(40 \%)$ \\
\hline HUMOR & $2(40 \%$ \\
\hline POSTURA & $2(40 \%)$ \\
\hline RESPIRAÇÃO & $1(20 \%)$ \\
\hline SONO & $2(40 \%)$ \\
\hline HÁBITO INTESTINAL & $----\cdots-\cdots---$ \\
\hline DISPOSIÇÃO & $2(40 \%)$ \\
\hline ALIMENTAÇÃOO & $1(20 \%)$ \\
\hline DORES FISICAS & $2(40 \%)$ \\
\hline DOENÇAS CRÔNICAS & $1(20 \%)$ \\
\hline
\end{tabular}

A segunda tabela mostra que a mesma tendência de resultados é observada no grupo que medita apenas uma vez por semana porém, o alcance é menor sugerindo que a prática disciplinada e diária (como ensinam as tradições) é necessária para que a plenitude dos resultados seja obtida.

Finalmente, observa-se que os resultados se reproduziram na segunda turma (realizada em 2011) sugerindo um caráter de reprodutibilidade ao procedimento, qualificando a meditação como um possível recurso a ser incorporado nas políticas publicas em Saúde Coletiva.(20)

Outra evidência, agora registrada no mundo corporativo, é de que, executivos que meditam começam a produzir projetos sustentáveis num processo de respeito ao colega de trabalho e parceria com a Natureza, ao mesmo tempo em que se tornam mais felizes, realizados e saudáveis!

A transdisciplinaridade é a atitude cientifica contemporânea que, reconhecendo a complexidade dos fenômenos assim como a multidimensionalidade da realidade, se dispõem a buscar soluções sustentáveis nos estratos mais sutis e causais (informacionais) da realidade o que possibilita encontrar situações de genuíno consenso coletivo, por meio da inclusão e 
respeito às diversidades, potencializando a utilização dos conhecimentos disciplinares de forma sinérgica e transformadora (21).

Esperamos que a disseminação desta atitude possa resgatar a dignidade da espécie humana recuperando a possibilidade de sua sobrevivência harmônica e pacifica no Planeta.

\section{REFERÊNCIAS BIBLIOGRÁFICAS}

ANAND, B; CHHINA, G. Investigations on Yogis claming to stop their Heartbeats. 1961 Indian Journal of Medical Research, 49: p. 90-94.

BENSON, H.; STARK, M. Medicina espiritual: o poder essencial da cura. Rio de Janeiro: Edições Campos, 1998 p. 9

BIGNARDI, F.A. C..; RAMOS, LR: PROTOCOLO DE MEDICINA TRANSDISCIPLINAR. Congresso Mundial de Transdisciplinaridade, 2005, Vila Velha, Brasil.

BIGNARDI, F. A. C..; OLIVEIRA M. C. G.; SILVEIRA, K. F.; BARROCO, M. F. C.; FERRAZ, D.; AMORIM, M. V. M. F.; OLIVEIRA, M. G. T.; FERRAZ, M. A. J.;

OLIVEIRA, R. A.; SANTANA, L.; CUNHA, M.S.; MULLER, W. ENSAIO CLÍNICO SOBRE OS EFEITOS DA MEDITAÇÃO APLICADA A UMA POPULAÇÃO IDOSA DE SÃO MATEUS. II Simpósio Internacional de Práticas contemplativas e Medicinas Tradicionais da UNIFESP, 2010.

BIGNARDI, F. A. C. et al. $\mathbf{1}^{\text {a }}$ Jornada de Transdisciplinaridade aplicada a Saúde da UNIFESP, 2010.

CAPRA F. As Conexões Ocultas: Ciência para uma vida sustentável. São Paulo: Ed. Cultrix, 2001.

DRAGER L. F.; PEDROSA, R. P.; DINIZ, P.M.; DIEGUES-SILVA L.; MARCONDES, B.; COUTO, R. B.; GIORGI, D. M.; KRIEGER, E. M.; LORENZI-FILHO; G. The Effects of Continuous Positive Airway Pressure on Prehypertension and Masked Hypertension in Men With Severe Obstructive Sleep Apnea. Hypertension. 2011 Jan 17. [Epub ahead of print] PubMed PMID: 21242462.

DURÁN-CANTOLLA J.; AIZPURU, F.; MONTSERRAT, J. M.; BALLESTER, E.; TERÁN-SANTOS, J, AGUIRREGOMOSCORTA, J. I.; GONZALEZ, M.; LLOBERES, P.; MASA, J. F.; DE LA PEÑA, M.; CARRIZO, S.; MAYOS, M.; BARBÉ, F. Spanish Sleep and Breathing Group. Continuous positive airway pressure as treatment for systemic hypertension in people with obstructive sleep apnoea: randomised controlled trial. BMJ. 2010 Nov 24; 341:c5991. doi: 10.1136/bmj.c5991. PubMed PMID: 21106625

GRANDIN T., Johnson C. Na Língua dos Bichos. Rio de Janeiro: Ed. Rocco. 2006.

GREEN, E. \&A. Beyond Biofeedback. New York: Dell, 1977.

GOSWAMI, Amit. A Janela Visionária. São Paulo: Ed. Cultrix, WHH, 2000, cap. 9.

LIMA-COSTA, M. F. F.; UCHÔA, E.; GUERRA, H. L.; FIRMO; J. O. A.; VIDIGAL, P. G.; BARRETO, S. M. The Bambuí Health and Ageing Study (BHAS). Methodological 
approach and preliminary results of a population-based cohort study of the elderly in Brazil. Revista de Saúde Pública, 2000b, 34: p. 126-135.

PASSARELLI, M.C.; JACOB-FILHO, W.; FIGUERAS, A. Adverse drug reactions in an elderly hospitalised population: inappropriate prescription is a leading cause. Drugs \& Aging. 2005; 22(9): p.767-7.

RAMOS, L. R. A mudança de Paradigma na saúde e o Conceito de Capacidade Funcional, Geriatria e Gerontologia, Guias de Medicina Ambulatorial e Hospitalar UNIFESP - EPM, 2005, Cap 1, p. 6.

: Two-year folow-up study of elderly residents in São Paulo, Brazil (Epidoso Project): methodology and preliminary results. Revista de Saúde pública, 33, (5): p.397-407, 1998.

RIANE, E. O Cálice e a Espada nosso futuro, nosso passado. São Paulo: Ed. Palas Athena, 2007.

SMITH, P. Linguagem Animal. São Paulo: Ed Mercuryo, 2004

SMITH, S. Advancing the mental connection. Ecletic Horse, issue 10. 2003.

TAYLOR, J. B. A Cientista que curou seu próprio Cérebro. Rio de Janeiro: Ediouro, 2008

GIMBUTAS, Marija. The Goddesses and Gods of Old Europe. Berkeley: University of CA Press, 1996.

NEVES, E. G.: Arqueologia da Amazônia. Rio de Janeiro: Jorge Zahar Editor, 2006. 